
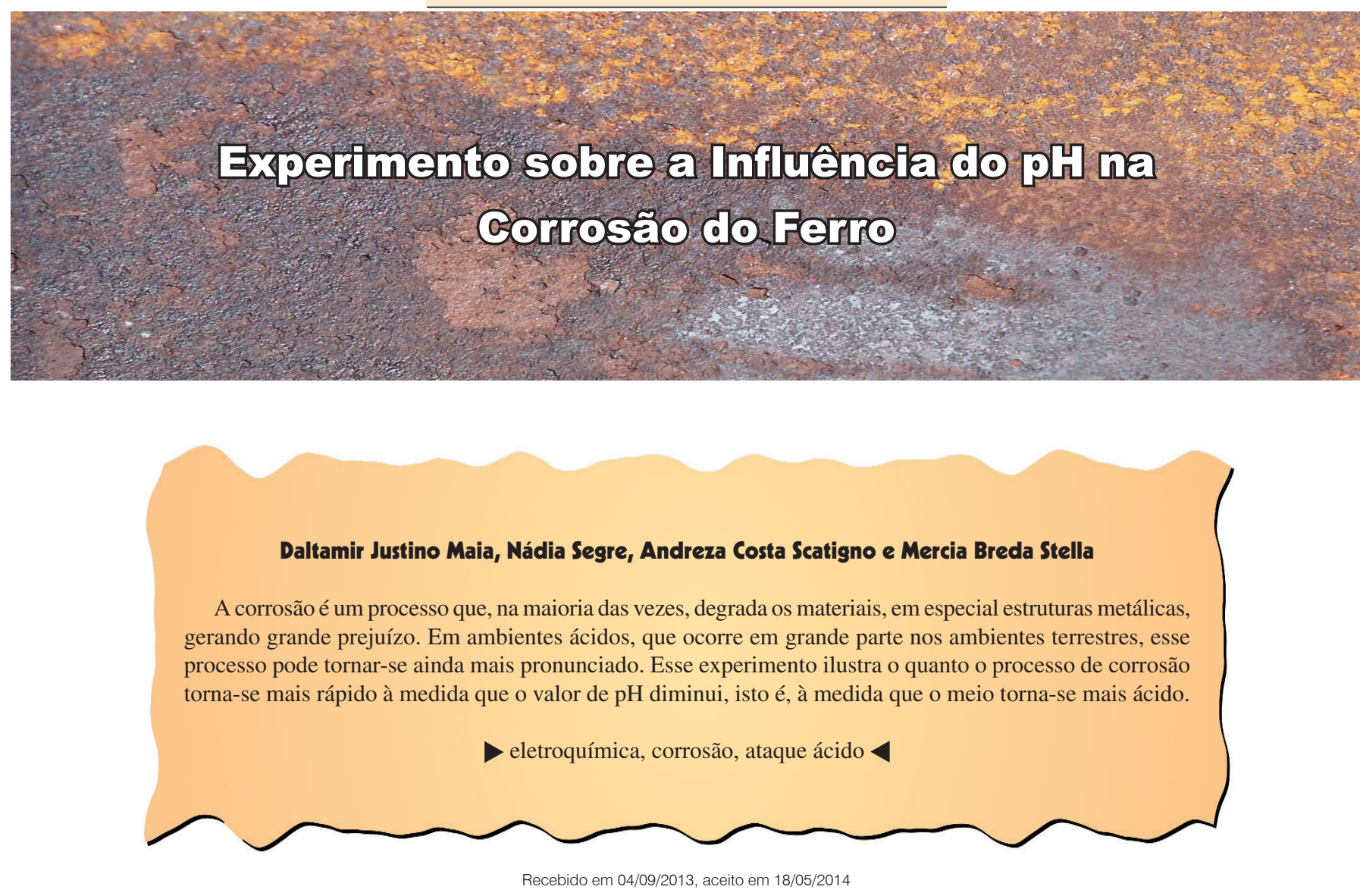

ב

m maior ou menor extensão, a maioria dos materiais experimenta algum tipo de interação com um grande número de ambientes diversos (Callister, 2008). Com frequência, tais interações comprometem a utilidade de um material como resultado da deterioração das suas propriedades mecânicas. Esses efeitos devem ser levados em consideração na hora da escolha do material mais adequado para um tipo de projeto a fim de garantir que os componentes não falhem prematuramente (Askeland; Phulé 2008).

Em seus projetos, um grande desafio para todos os profissionais que empregam metais - além de outros tipos de materiais tais como concretos e plásticos - é impedir que estes sofram ataques corrosivos/destrutivos (Shackelford, 2008). Como a prevenção completa é muito difícil, as perdas devem ser minimizadas. Os mecanismos de deterioração são muito diversos para os diferentes tipos de materiais, mas, para os metais, existe uma efetiva perda de material, seja por dissolução (corrosão) ou pela formação de uma incrustação ou película de material não metálico (oxidação).

A corrosão pode ser definida como o ataque destrutivo e não intencional sofrido por um metal, que normalmente

\footnotetext{
A seção "Experimentação no ensino de Química" descreve experimentos cuja implementação e interpretação contribuem para a construção de conceitos científicos por parte dos alunos. Os materiais e reagentes usados são facilmente encontráveis, permitindo a realização dos experimentos em qualquer escola.
}

começa na superfície do material. O problema da corrosão metálica apresenta proporções significativas. Em termos econômicos, foi estimado que aproximadamente $5 \%$ da receita de um país industrializado são gastos na prevenção da corrosão e na manutenção ou na substituição de produtos perdidos ou contaminados como resultado das reações de corrosão. Isso sem falar dos acidentes ou perdas de vidas humanas provocadas por contaminações, poluição e falta de segurança dos equipamentos (Merçon et al., 2004).

Uma vez que a corrosão é provocada por uma reação química, a velocidade com a qual esta se processa dependerá, até certo ponto, da temperatura e da concentração das espécies envolvidas. Outros fatores, tais como os esforços mecânicos e a erosão, também podem contribuir para a corrosão (Smith, 1998).

A maioria dos metais é corroída de algum modo por efeito da água e da atmosfera, portanto, as espécies presentes na água (principalmente espécies iônicas) acabam tendo uma importância muito grande nos processos de deterioração dos metais. Por exemplo, a acidez devido à presença de espécies na água, que pode ser medida por meio dos valores de $\mathrm{pHs}$, tem um papel fundamental na corrosão dos metais.

\section{Atmosfera oxidativa}

O ambiente terrestre atual é naturalmente oxidativo 
devido à presença de oxigênio e água $\left(\mathrm{O}_{2}\right.$ e $\left.\mathrm{H}_{2} \mathrm{O}\right)$, portanto, espontaneamente, os metais tendem a sofrer oxidação quando em contato com essas substâncias. Além disso, à medida que substâncias ácidas estão presentes na água, a velocidade de oxidação dos metais tende a aumentar. A chuva é normalmente ácida (valores de $\mathrm{pH}$ menor que 7) devido à presença de ácido carbônico (formado pela reação entre o gás carbônico e a água: $\mathrm{CO}_{2}+\mathrm{H}_{2} \mathrm{O} \rightarrow \mathrm{H}_{2} \mathrm{CO}_{3}$ ). Quando a água está saturada de gás carbônico, o valor de $\mathrm{pH}$ fica em torno de 5,6. Portanto, até valor de $\mathrm{pH}=5,6$, não usamos o termo chuva ácida para esse tipo de precipitação (Maia et al., 2005).

No entanto, a presença de poluentes tais como óxidos de enxofre $\left(\mathrm{SO}_{2}\right.$ e $\left.\mathrm{SO}_{3}\right)$ e de nitrogênio $\left(\mathrm{N}_{2} \mathrm{O}, \mathrm{NO}\right.$ e $\left.\mathrm{NO}_{2}\right)$, presentes na atmosfera, principalmente em atmosferas poluídas, forma ácidos, aumentando a acidez da chuva. Existem registros de chuvas com valor de $\mathrm{pH}$ em torno de 2,0, lembrando que a escala de pH é logarítmica, isto é, para cada unidade de $\mathrm{pH}$, temos uma variação de 10 unidades na concentração de $\mathrm{H}^{+}$. Isso significa que, de 5,6 para 2, temos um aumento de quase 4000 vezes na concentração de $\mathrm{H}^{+}$ (Maia et al., 2005).

\section{Considerações eletroquímicas}

Para os materiais metálicos, o processo de corrosão é normalmente um processo eletroquímico, isto é, uma reação química em que existe uma transferência de elétrons de uma espécie química para outra (processo denominado de oxidorredução). Caracteristicamente, os átomos metálicos têm seus elétrons retirados, no que é chamada uma reação de oxidação. A quantidade de elétrons retirada de um determinado metal é uma característica dele e pode variar de um metal para outro. Por exemplo, para a prata, o ferro e o alumínio, podemos ter os seguintes processos de oxidação:

$$
\begin{aligned}
& \mathrm{Ag} \rightarrow \mathrm{Ag}^{+}+1 \mathrm{e}^{-} \\
& \mathrm{Fe} \rightarrow \mathrm{Fe}^{2+}+2 \mathrm{e}^{-} \\
& \mathrm{Al} \rightarrow \mathrm{Al}^{3+}+3 \mathrm{e}^{-}
\end{aligned}
$$

As espécies que retiram os elétrons do metal sofrem um processo denominado de redução. Essas espécies, ao retirarem elétrons e provocarem a oxidação de outras, são denominadas de agentes oxidantes. O ar atmosférico, devido à presença de gás oxigênio (compõe $20 \%$ do ar seco) e água (presente na forma de vapor), é um ambiente propício à oxidação.

Por exemplo, ligas de aço, cujo principal metal constituinte é o ferro, quando expostas ao oxigênio e à água $\left(\mathrm{O}_{2} \mathrm{e}\right.$ $\mathrm{H}_{2} \mathrm{O}$ ), sofrem corrosão com o passar do tempo e formam a ferrugem, que é uma mistura de óxidos e hidróxidos de ferro hidratados. Dependendo do valor de $\mathrm{pH}$ e das concentrações de gás oxigênio e água, essa mistura tem proporções diferentes, principalmente, das seguintes espécies: $\mathrm{Fe}_{3} \mathrm{O}_{4} / \mathrm{Fe}_{2} \mathrm{O}_{3}$. $\mathrm{H}_{2} \mathrm{O} / \mathrm{Fe}(\mathrm{OH})_{2} / \mathrm{Fe}(\mathrm{OH})_{3}$. Quanto maior o teor de $\mathrm{Fe}_{3} \mathrm{O}_{4}$ (preto), mais percebemos que o objeto em questão está sofrendo corrosão. Quanto mais $\mathrm{Fe}_{2} \mathrm{O}_{3}$. $\mathrm{H}_{2} \mathrm{O}$, maior a intensidade da coloração alaranjada ou castanho avermelhada. $\mathrm{O} \mathrm{Fe}(\mathrm{OH})_{2}$ puro é branco, mas em contato com oxigênio, torna-se verde. $\mathrm{O} \mathrm{Fe}(\mathrm{OH})_{3}$ puro é marrom. Portanto, dependendo da predominância de cada uma dessas espécies, temos uma tonalidade mais pronunciada do produto da corrosão.

\section{O ambiente ácido acelera o processo de oxidação}

Quanto mais ácido o ambiente (maior concentração de íons $\mathrm{H}^{+}$), maior a velocidade de oxidação das estruturas metálicas. Em um ambiente ácido, o processo predominante de oxidação pode ser representado da seguinte maneira:

Redução do $\mathrm{O}_{2}$ por ação dos íons hidrogênio:

$$
\mathrm{O}_{2}+4 \mathrm{H}^{+}+4 \mathrm{e}^{-} \rightarrow 2 \mathrm{H}_{2} \mathrm{O}
$$

Oxidação dos íons metálicos:

$$
\mathrm{Fe} \rightarrow \mathrm{Fe}^{3+}+3 \mathrm{e}^{-}
$$

Quanto mais ácido um meio, maior a diferença entre a concentração de íons $\mathrm{H}^{+}$em relação aos íons $\mathrm{OH}^{-}$(meio ácido: $\left.\left[\mathrm{H}^{+}\right]>\left[\mathrm{OH}^{-}\right]\right)$. Nesse caso, o produto formado será preferencialmente óxido de ferro $\left(\mathrm{Fe}_{3} \mathrm{O}_{4}\right.$ - coloração preta).

\section{Efeito do pH}

O efeito da acidez na velocidade de corrosão do ferro, em água aerada e em temperatura ambiente, pode ser verificado na Figura 1 (Gentil 2011).

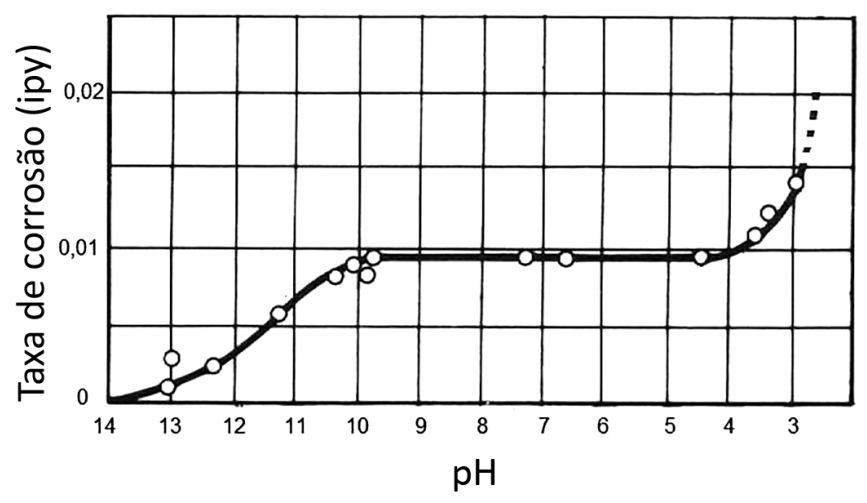

Figura 1: Correlação entre o pH e a taxa de corrosão do ferro (Gentil, 2011).

Verifica-se uma interferência cada vez mais positiva (aumento da corrosão) quando temos valores de pHs abaixo de 4. Para valores de pHs entre 4 e 10, a taxa de corrosão independe do $\mathrm{pH}$ e depende somente da rapidez com que o oxigênio difunde para a superfície metálica. Quando os valores de pHs são maiores que 10, esse aumento mais pronunciado da alcalinidade do meio faz com que a taxa de corrosão diminua, pois o ferro se torna passivo em presença de álcalis e oxigênio dissolvido. 


\section{Experimento}

\section{Reagentes e materiais}

$100 \mathrm{~mL}$ de ácido clorídrico $(\mathrm{HCl})$ 0,1 mol L-1 (pode ser encontrado na forma concentrada em lojas de construção como ácido muriático); $100 \mathrm{~mL}$ de ácido nítrico $\left(\mathrm{HNO}_{3}\right)$ 0,1 $\mathrm{mol} \mathrm{L}^{-1} ; 1$ pacote de esponja de aço; água destilada; 14 balões volumétricos de $100 \mathrm{~mL}$; béqueres; balança; pipeta graduada $(10 \mathrm{~mL})$; pisseta; proveta $(100 \mathrm{~mL})$; pera de borracha; bomba de aspergir.

\section{Preparo das soluções de $\mathrm{HCl}$ e $\mathrm{HNO}_{3}$}

A partir da solução $0,1 \mathrm{~mol} \mathrm{~L}^{-1}(\mathrm{pH}=1)$ de ácido clorídrico, por meio de diluições sucessivas, foram preparadas soluções com valores de $\mathrm{pH}=2,3,4,5,6$ e 7 . O mesmo procedimento foi feito na preparação das soluções de ácido nítrico. A partir da solução estoque desse ácido $\left(0,1 \mathrm{~mol} \mathrm{~L}^{-1}\right.$ $-\mathrm{pH}=1)$, prepararam-se as outras soluções com valores de $\mathrm{pH}=2,3,4,5,6$ e 7 .

\section{Procedimento}

Pesar 14 amostras de esponjas de aço. Se possível, ajustar para que elas tenham aproximadamente a mesma massa para melhor comparação - cada esponja pode ser cortada em dois pedaços.

Colocar cada esponja sobre um anteparo (placa de petri ou similar). Colocar a solução de ácido clorídrico $(\mathrm{pH}=1)$ na bomba de aspergir, regular a saída e borrifar 10 vezes sobre a esponja. Lavar a bomba de aspergir e repetir o procedimento para cada uma das outras soluções $(\mathrm{pH}=2,3,4,5,6$ e 7). Repetir o mesmo procedimento utilizando as soluções de ácido nítrico.

Deixe as 14 amostras em repouso durante 24 horas. Esse período assegura que a esponja fique completamente seca, garantindo que o aumento de massa é devido basicamente ao processo de oxidação. Depois, verifique visualmente o que ocorreu com cada uma das amostras e pese cada uma comparando com a massa inicial. $\mathrm{O}$ ácido pode ser descartado na pia, desde que esteja diluído ou neutralizado.

\section{Resultados}

Após um dia de oxidação (24 horas), podemos verificar visualmente que o processo oxidativo é mais eficaz quando os valores de pH estão abaixo de 4 (meios mais ácidos): maior taxa de corrosão tanto para as amostras em contato com soluções de ácido clorídrico quanto nas amostras em contato com soluções de ácido nítrico. As Figuras 2 e 3 ilustram as amostras de esponjas de aço oxidadas. Nos dois casos, as corrosões em soluções com valores de $\mathrm{pH} \leq 3$ foram mais intensas, enquanto em valores de $\mathrm{pH}=4,5,6$ e 7 , a diferença não foi tão evidente (praticamente constante). Visualmente já é possível observar que o processo oxidativo é bem mais intenso nas amostras com valores de $\mathrm{pH}=1$, de maior acidez, tanto para o ácido clorídrico quanto para o ácido nítrico. Isso indica que o maior efeito oxidativo ocorre pela presença dos

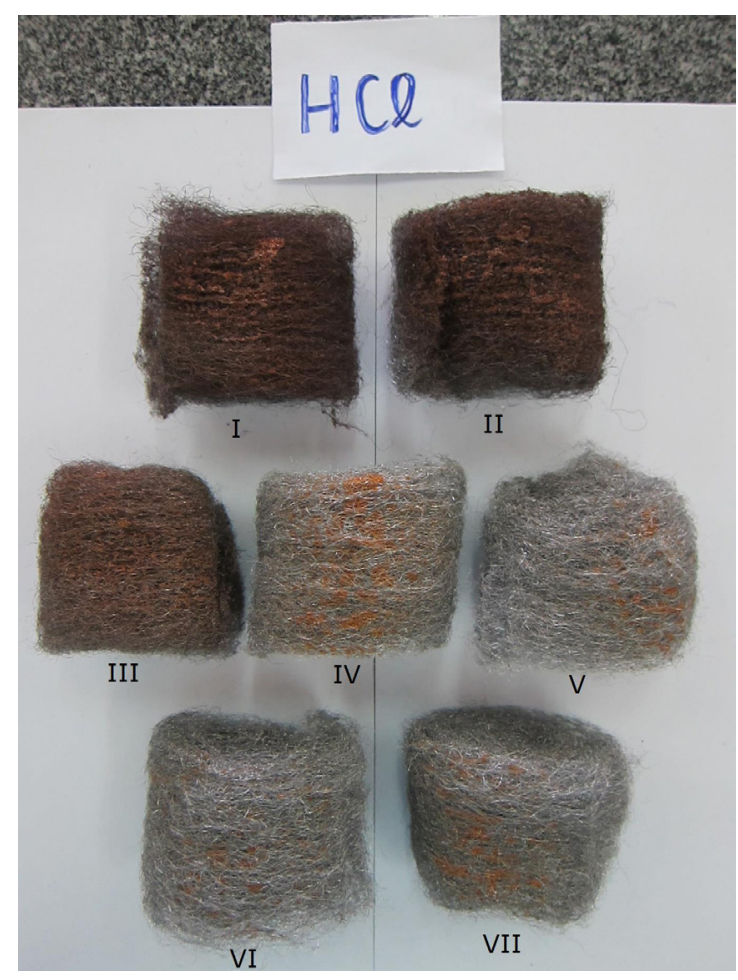

Figura 2: Amostras de esponja de aço após um dia de oxidação frente às soluções de ácido clorídrico. I) solução 1,0 × $10^{-1} \mathrm{~mol} \mathrm{~L}^{-1}$ $(\mathrm{pH}=1)$; II) solução $1,0 \times 10^{-2} \mathrm{~mol} \mathrm{~L}^{-1}(\mathrm{pH}=2)$; III) solução $\left.1,0 \times 10^{-3} \mathrm{~mol} \mathrm{~L}^{-1}(\mathrm{pH}=3) ; \mathrm{IV}\right)$ solução $1,0 \times 10^{-4} \mathrm{~mol} \mathrm{~L}^{-1}(\mathrm{pH}=4)$; V) solução $1,0 \times 10^{-5} \mathrm{~mol} \mathrm{~L}^{-1}(\mathrm{pH}=5)$; solução $1,0 \times 10^{-6} \mathrm{~mol} \mathrm{~L}^{-1}$

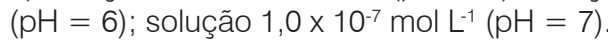

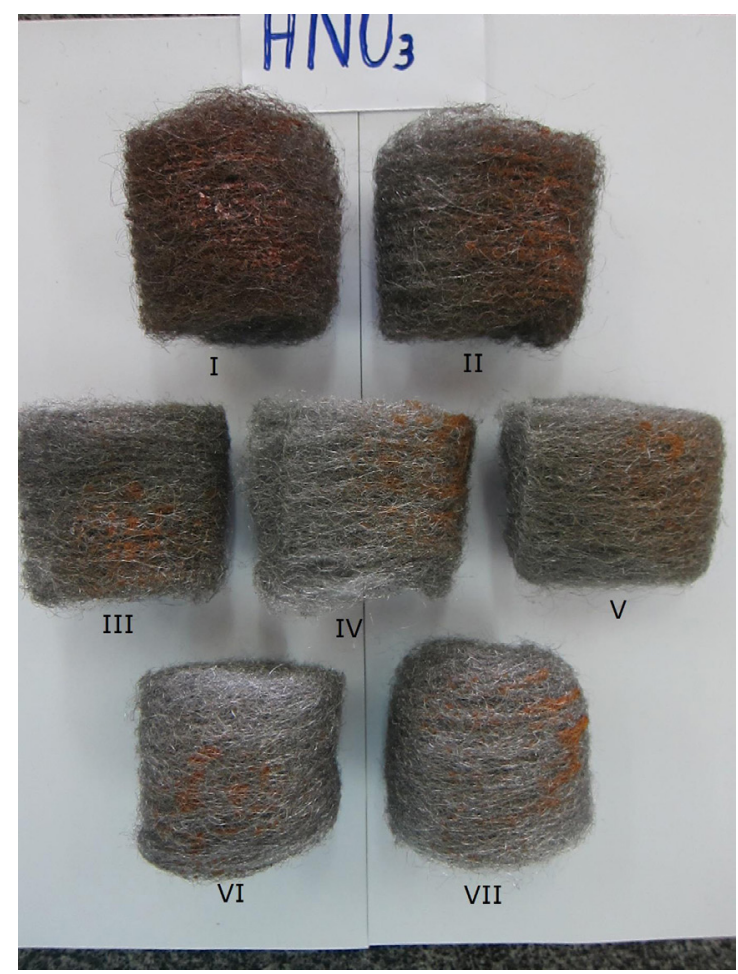

Figura 3: Amostras de esponja de aço após um dia de oxidação frente às soluções de ácido nítrico. I) solução $1,0 \times 10^{-1} \mathrm{~mol} \mathrm{~L}^{-1}$ $(\mathrm{pH}=1)$; II) solução $1,0 \times 10^{-2} \mathrm{~mol} \mathrm{~L}^{-1}(\mathrm{pH}=2)$; III) solução $\left.1,0 \times 10^{-3} \mathrm{~mol} \mathrm{~L}^{-1}(\mathrm{pH}=3) ; \mathrm{IV}\right)$ solução $1,0 \times 10^{-4} \mathrm{~mol} \mathrm{~L}^{-1}(\mathrm{pH}=4) ;$ V) solução $1,0 \times 10^{-5} \mathrm{~mol} \mathrm{~L}^{-1}(\mathrm{pH}=5)$; solução $1,0 \times 10^{-6} \mathrm{~mol} \mathrm{~L}^{-1}$ $(\mathrm{pH}=6)$; solução $1,0 \times 10^{-7} \mathrm{~mol} \mathrm{~L}^{-1}(\mathrm{pH}=7)$. 
Tabela 1: Massas das esponjas de aço antes e após um dia de oxidação frente às soluções de ácido clorídrico.

\begin{tabular}{lccccccc}
\hline & $\begin{array}{c}\text { Amostra I } \\
\mathrm{pH}=1\end{array}$ & $\begin{array}{c}\text { Amostra II } \\
\mathrm{pH}=2\end{array}$ & $\begin{array}{c}\text { Amostra III } \\
\mathrm{pH}=3\end{array}$ & $\begin{array}{c}\text { Amostra IV } \\
\mathrm{pH}=4\end{array}$ & $\begin{array}{c}\text { Amostra V } \\
\mathrm{pH}=5\end{array}$ & $\begin{array}{c}\text { Amostra VI } \\
\mathrm{pH}=6\end{array}$ & $\begin{array}{c}\text { Amostra VII } \\
\mathrm{pH}=7\end{array}$ \\
\hline Massa inicial $(\mathrm{g})$ & 3,858 & 3,915 & 3,608 & 3,438 & 3,391 & 3,313 & 3,458 \\
Massa final (g) & 4,076 & 4,102 & 3,675 & 3,450 & 3,397 & 3,317 & 3,464 \\
Aumento de massa (g) & 0,218 & 0,187 & 0,067 & 0,012 & 0,006 & 0,004 & 0,006 \\
\% do aumento de massa & $5,35 \%$ & $4,56 \%$ & $1,82 \%$ & $0,35 \%$ & $0,18 \%$ & $0,12 \%$ & $0,17 \%$ \\
\hline
\end{tabular}

Tabela 2: Massas das esponjas de aço antes e após um dia de oxidação frente às soluções de ácido nítrico.

\begin{tabular}{lccccccc}
\hline & $\begin{array}{c}\text { Amostra I } \\
\mathrm{pH}=1\end{array}$ & $\begin{array}{c}\text { Amostra II } \\
\mathrm{pH}=2\end{array}$ & $\begin{array}{c}\text { Amostra III } \\
\mathrm{pH}=3\end{array}$ & $\begin{array}{c}\text { Amostra IV } \\
\mathrm{pH}=4\end{array}$ & $\begin{array}{c}\text { Amostra V } \\
\mathrm{pH}=5\end{array}$ & $\begin{array}{c}\text { Amostra VI } \\
\mathrm{pH}=6\end{array}$ & $\begin{array}{c}\text { Amostra VII } \\
\mathrm{pH}=7\end{array}$ \\
\hline Massa inicial $(\mathrm{g})$ & 3,921 & 3,714 & 3,740 & 3,140 & 3,679 & 3,695 & 3,543 \\
Massa final $(\mathrm{g})$ & 4,102 & 3,852 & 3,783 & 3,147 & 3,684 & 3,697 & 3,549 \\
Aumento de massa $(\mathrm{g})$ & 0,181 & 0,138 & 0,043 & 0,007 & 0,005 & 0,002 & 0,006 \\
\% do aumento de massa & $4,41 \%$ & $3,58 \%$ & $1,14 \%$ & $0,22 \%$ & $0,14 \%$ & $0,05 \%$ & $0,17 \%$ \\
\hline
\end{tabular}

íons $\mathrm{H}^{+}$independente do ácido utilizado.

Em soluções com elevada concentração de íons $\mathrm{H}^{+}$(valores de $\mathrm{pH}$ abaixo de 4$)$, ocorre redução destes $\left(2 \mathrm{H}^{+}+2 \mathrm{e}^{-} \rightarrow\right.$ $\mathrm{H}_{2}$ ) e consequente oxidação do ferro (Callister, 2008). No entanto, quando as soluções são menos concentradas e com a presença de oxigênio, ocorre preferencialmente o seguinte processo redutivo: $\mathrm{O}_{2}+4 \mathrm{H}^{+}+4 \mathrm{e}^{-} \rightarrow 2 \mathrm{H}_{2} \mathrm{O}$. A velocidade de corrosão diminui entre valores de pHs 4 e 7 , pois esta depende somente da rapidez com que o oxigênio difunde para a superfície metálica.

As Tabelas 1 e 2 apresentam as massas das esponjas antes e depois de um dia de oxidação, a variação de massa ocorrida e a porcentagem de aumento de massa. $\mathrm{O}$ aumento de massa é devido à ferrugem que se forma, principalmente na forma de hidróxido de ferro III $\mathrm{Fe}(\mathrm{OH})_{3}$, pois a cor predominante, conforme pode ser visto pelas Figuras 2 e 3, é amarronzada. $\mathrm{O}$ maior aumento de massa, à medida que $\mathrm{o}$ valor de $\mathrm{pH}$ diminui, é reflexo da maior taxa de corrosão da esponja de aço. Esse aumento é mais pronunciado à medida que o meio torna-se mais ácido.

Utilizando os valores de porcentagem do aumento de massa em função do valor de $\mathrm{pH}$, podemos construir um gráfico similar ao da Figura 1, tanto para o $\mathrm{HCl}$ quanto para o $\mathrm{HNO}_{3}$, e comprovar a dependência da corrosão com a concentração de $\mathrm{H}^{+}$. Esses resultados estão presentes no Gráfico 1 .

O aumento na massa das amostras não fere a Lei de Conservação das Massas (Lavoisier), pois esse aumento é em decorrência da incorporação de hidrogênio e oxigênio na amostra por meio do processo de oxidação. Podemos calcular

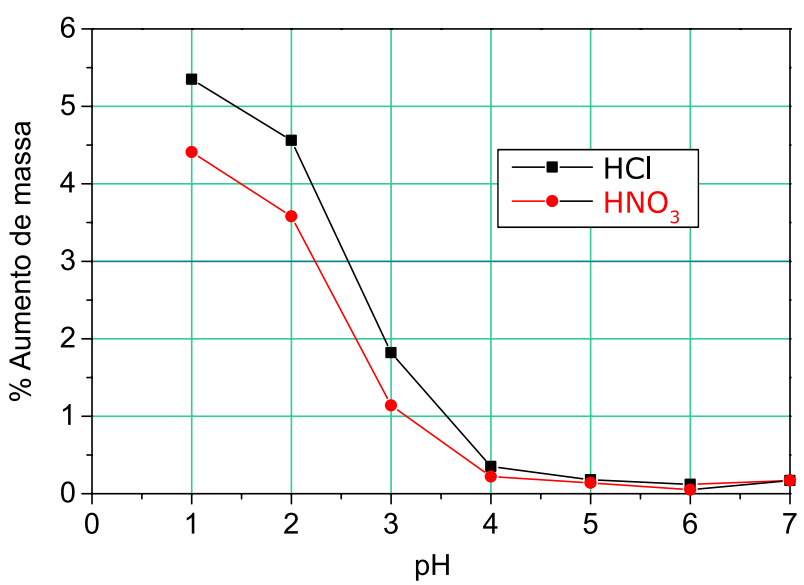

Gráfico 1: Correlação entre o pH e a corrosão (porcentagem no aumento de massa) do ferro em soluções aquosas de $\mathrm{HCl}$ e de $\mathrm{HNO}_{3}$.

a quantidade de ferro que sofreu oxidação, considerando que todo aumento de massa foi em decorrência da reação:

$$
\underset{56 \mathrm{~g}}{\stackrel{\mathrm{O}_{2} / \mathrm{H}_{2} \mathrm{O}}{\longrightarrow}} \underset{107 \mathrm{~g} \text { (aumento de } 51 \mathrm{~g} \text { ) }}{\mathrm{Fe}(\mathrm{OH})_{3}}
$$

Nesse caso, as esponjas ficaram preferencialmente na cor marrom. Para cada aumento de massa de $51 \mathrm{~g}$, ocorre a oxidação de $56 \mathrm{~g}$ de ferro $(1 \mathrm{~mol})$. Desse modo, podemos determinar a quantidade de matéria que oxidou em cada amostra. As Tabelas 3 e 4 apresentam os valores de massa para cada amostra analisada.

Tabela 3: Massa e quantidade de matéria de ferro que sofreu oxidação após um dia de contato com soluções de ácido clorídrico.

\begin{tabular}{lccccccc}
\hline & $\begin{array}{c}\text { Amostra I } \\
\mathrm{pH}=1\end{array}$ & $\begin{array}{c}\text { Amostra II } \\
\mathrm{pH}=2\end{array}$ & $\begin{array}{c}\text { Amostra III } \\
\mathrm{pH}=3\end{array}$ & $\begin{array}{c}\text { Amostra IV } \\
\mathrm{pH}=4\end{array}$ & $\begin{array}{c}\text { Amostra V } \\
\mathrm{pH}=5\end{array}$ & $\begin{array}{c}\text { Amostra VI } \\
\mathrm{pH}=6\end{array}$ & $\begin{array}{c}\text { Amostra VII } \\
\mathrm{pH}=7\end{array}$ \\
\hline Aumento de massa (g) & 0,218 & 0,187 & 0,067 & 0,012 & 0,006 & 0,004 & 0,006 \\
Massa de ferro que oxidou (g) & 0,239 & 0,205 & 0,074 & 0,013 & 0,007 & 0,004 & 0,007 \\
Quantidade de matéria (mol) & $4,27 \times 10^{-3}$ & $3,66 \times 10^{-3}$ & $1,32 \times 10^{-3}$ & $2,32 \times 10^{-4}$ & $1,25 \times 10^{-4}$ & $7,14 \times 10^{-5}$ & $1,25 \times 10^{-4}$ \\
\hline
\end{tabular}


Tabela 4: Massa e quantidade de matéria de ferro que sofreu oxidação após um dia de contato com soluções de ácido nítrico.

\begin{tabular}{lccccccc}
\hline & $\begin{array}{c}\text { Amostra I } \\
\mathrm{pH}=1\end{array}$ & $\begin{array}{c}\text { Amostra II } \\
\mathrm{pH}=2\end{array}$ & $\begin{array}{c}\text { Amostra III } \\
\mathrm{pH}=3\end{array}$ & $\begin{array}{c}\text { Amostra IV } \\
\mathrm{pH}=4\end{array}$ & $\begin{array}{c}\text { Amostra V } \\
\mathrm{pH}=5\end{array}$ & $\begin{array}{c}\text { Amostra VI } \\
\mathrm{pH}=6\end{array}$ & $\begin{array}{c}\text { Amostra VII } \\
\mathrm{pH}=7\end{array}$ \\
\hline Aumento de massa (g) & 0,181 & 0,138 & 0,043 & 0,007 & 0,005 & 0,002 & 0,006 \\
Massa de ferro que oxidou (g) & 0,199 & 0,152 & 0,047 & 0,008 & 0,005 & 0,002 & 0,007 \\
Quantidade de matéria (mol) & $3,55 \times 10^{-3}$ & $2,71 \times 10^{-3}$ & $8,39 \times 10^{-4}$ & $1,4 \times 10^{-4}$ & $8,93 \times 10^{-5}$ & $3,57 \times 10^{-5}$ & $1,25 \times 10^{-4}$ \\
\hline
\end{tabular}

\section{Conclusões}

O experimento comprova o aumento da taxa de corrosão em materiais ferrosos em soluções cada vez mais ácidas, principalmente quando os valores de $\mathrm{pHs}$ tornam-se menores que 4. Isso explica porque os problemas de corrosão de estruturas metálicas em aço tendem a ser mais intensos em algumas regiões mais industrializadas e poluídas (devido à formação da chuva ácida).

A porcentagem e o comportamento do metal frente à corrosão é praticamente a mesma, independente do tipo de ácido, comprovando que o mais importante no aumento da corrosão é a concentração de íons $\mathrm{H}^{+}$no meio.

Esse experimento, simples de ser realizado com materiais de fácil acesso e descarte, demonstra a influência da acidez na corrosão de um material metálico, podendo auxiliar no aprendizado dos conceitos de oxidorredução. Os alunos podem também correlacionar a problemática da corrosão e a influência do meio ambiente (poluição) nesse processo, principalmente no que diz respeito à chuva ácida.

\section{Referências}

ASKELAND, D.R.; PHULÉ, P.P. Ciência e engenharia dos materiais. São Paulo: Cengage Learning, 2008.

CALLISTER JR., W.D. Fundamentos da ciência e engenharia de materiais. 2. ed. Rio de Janeiro: LTC, 2008.

GENTIL, V. Corrosão. 6. ed. Rio de Janeiro: LTC, 2011.

MAIA, D.J.; GAZOTTI, W.A.; CANELA, M.C.; SIQUEIRA, A.E. Chuva ácida: um experimento para introduzir conceitos de equilíbrio químico e acidez no ensino médio. Química Nova na Escola, n. 21, p. 44-46, 2005.

MERCON, F.; GUIMARÃES, P.I.C.; MAINER, F.B. Corrosão: um exemplo usual de fenômeno químico. Química Nova na
Também é possível trabalhar os conceitos da Lei de Conservação das Massas de Lavoisier com essas amostras e chamar a atenção para o aumento de massa nas esponjas, que foi em decorrência da incorporação de hidrogênio e oxigênio na formação da ferrugem.

Daltamir Justino Maia (daltamir.maia@gmail.com), graduado em química pela UNICAMP, mestre e doutor pela UNICAMP, é professor titular no grupo Anhanguera Educacional. Campinas, SP - BR. Nádia Segre (nsegre@yahoo. com), bacharel em Química pela UNICAMP, mestre em Físico-Química e Doutorado em Ciências pelo Instituto de Química da UNICAMP, é professora titular na Faculdade Anhanguera. Sorocaba, SP - BR. Andreza Costa Scatigno (decost@ig.com.br), graduada em Química e em Química Tecnológica pela UNESP-Araraquara, mestre em Biotecnologia pela UNESP-Araraquara, doutora em Ciências pela USP, é pesquisadora e professora do grupo Anhanguera Educacional, da FACENS e do Pitágoras. Sorocaba, SP - BR. Mercia Breda Stella (merciabreda@uol.com.br),graduada em biomedicina pela UMC e nutrição pela FESB, mestre em Ciências - Bioquímica pelo Instituto de Química - USP e Doutora em Bioquímica pelo Instituto de Biologia - UNICAMP, é professora titular de Bioquímica e Biofísica da Faculdade Anhanguera de Campinas - FAC III e titular e regente de Bioquímica e Biofísica da Faculdade de Medicina de Jundiaí. Campinas, SP - BR.

Escola, n. 19, p. 11-14, 2004.

SHACKELFORD, J.F. Ciência dos materiais. 6. ed. São Paulo: Pearson, 2008.

SMITH, W.F. Princípios de ciência e engenharia dos materiais. São Paulo: McGraw-Hill, 1998.

\section{Para saber mais}

DUTRA, A.C.; NUNES, L.P. Proteção catódica - técnica de combate à corrosão. Rio de Janeiro: Técnica, 1987.

MAIA, D.J.; BIANCHI, J.C.A. Fundamentos de química. São Paulo: Pearson, 2007.

Abstract: Experiment about the influence of pH on iron corrosion. Corrosion is a process often responsible for materials degradation, in particular for steel structures, causing significant damages. In acidic environments, which is very common in terrestrial environments, this degradation process can become even more pronounced. The experiment proposed in this work illustrates that the lower the $\mathrm{pH}$ the higher the etching process.

Keywords: electrochemical, corrosion, acid attack 